\title{
Quantitative profiling of oxylipins through comprehensive LC-MS/MS analysis: application in cardiac surgery
}

\author{
Katrin Strassburg • Annemarie M. L. Huijbrechts • Kirsten A. Kortekaas • \\ Jan H. Lindeman • Theresa L. Pedersen • Adrie Dane • Ruud Berger • \\ Arjan Brenkman • Thomas Hankemeier • John van Duynhoven • Eric Kalkhoven • \\ John W. Newman • Rob J. Vreeken \\ Received: 2 May 2012 /Revised: 21 June 2012 / Accepted: 22 June 2012 /Published online: 20 July 2012 \\ (C) The Author(s) 2012. This article is published with open access at Springerlink.com
}

\begin{abstract}
Oxylipins, including eicosanoids, affect a broad range of biological processes, such as the initiation and resolution of inflammation. These compounds, also referred to as lipid mediators, are (non-) enzymatically generated by oxidation of polyunsaturated fatty acids such as arachidonic acid (AA). A plethora of lipid mediators exist which makes the development of generic analytical methods challenging. Here we developed a robust and sensitive targeted analysis platform
\end{abstract}

Katrin Strassburg, Annemarie M. L. Huijbrechts, Erik Kalkhoven, John W. Newman and Rob J. Vreeken have contributed equally to the work published in this paper.

Electronic supplementary material The online version of this article (doi:10.1007/s00216-012-6226-x) contains supplementary material, which is available to authorized users.

K. Strassburg $\cdot$ A. Dane $\cdot$ T. Hankemeier $\cdot$ R. J. Vreeken

Leiden Amsterdam Centre for Drug Research, Leiden University,

Einsteinweg 55,

2300 RA Leiden, The Netherlands

A. M. L. Huijbrechts · R. Berger · A. Brenkman · E. Kalkhoven Department of Metabolic and Endocrine Diseases,

University Medical Centre Utrecht,

Utrecht, The Netherlands

K. Strassburg • A. M. L. Huijbrechts · A. Dane • R. Berger •

A. Brenkman · T. Hankemeier · J. van Duynhoven •

E. Kalkhoven $\cdot$ R. J. Vreeken

Netherlands Metabolomics Centre,

Einsteinweg 55,

2300 RA Leiden, The Netherlands

\section{K. A. Kortekaas}

Department of Cardiothoracic Surgery,

Leiden University Medical Center,

Albinusdreef 2-Dialyse BO-P,

2333 ZA Leiden, The Netherlands

\section{J. H. Lindeman}

Department of General Surgery,

Leiden University Medical Center,

Leiden, The Netherlands for oxylipins and applied it in a biological setting, using high performance liquid chromatography coupled to tandem mass spectrometry (HPLC-MS/MS) operated in dynamic multiple reaction monitoring (dMRM). Besides the well-described AA metabolites, oxylipins derived from linoleic acid, dihomo- $\gamma$ linolenic acid, $\alpha$-linolenic acid, eicosapentaenoic acid and docosahexaenoic acid were included. Our comprehensive platform allows the quantitative evaluation of approximately

T. L. Pedersen $\cdot$ J. W. Newman

USDA-ARS Western Human Nutrition Research Center,

430 West Health Sciences,

Davis, CA, USA

J. W. Newman

Department of Nutrition, University of California,

430 West Health Sciences,

Davis, CA, USA

J. van Duynhoven

Unilever Research and Development, Olivier van Noortlaan 120,

3133 AT Vlaardingen, The Netherlands

J. van Duynhoven

Laboratory of Biophysics, Wageningen University,

Dreijenlaan 3,

6703 HA Wageningen, The Netherlands

R. J. Vreeken $(\bowtie)$

Department of Analytical BioSciences, Leiden

Amsterdam Centre for Drug Research, Leiden University,

Einsteinweg 55,

2300 RA Leiden, The Netherlands

e-mail: vreekenrj@lacdr.leidenuniv.nl 
100 oxylipins down to low nanomolar levels. Applicability of the analytical platform was demonstrated by analyzing plasma samples of patients undergoing cardiac surgery. Altered levels of some of the oxylipins, especially in certain monohydroxy fatty acids such as 12-HETE and 12-HEPE, were observed in samples collected before and $24 \mathrm{~h}$ after cardiac surgery. These findings indicate that this generic oxylipin profiling platform can be applied broadly to study these highly bioactive compounds in relation to human disease.

Keywords HPLC-MS/MS · dMRM · 12-HETE · 12-HEPE

\section{Introduction}

Oxylipins are oxygenated metabolites derived from polyunsaturated fatty acids (PUFAs) such as arachidonic acid (AA), linoleic acid (LA), eicosapentaenoic acid (EPA), docosahexaenoic acid (DHA), and dihomo- $\gamma$-linolenic acid (DGLA). The oxygenated 20-carbon products, collectively referred to as eicosanoids, are the best-described lipid mediators. Among their many functions, these lipids are potent inflammatory modulators and thus have been associated with responses to cardiovascular diseases, host defense, tissue injury, and surgical intervention [1-3].

Through both, enzymatic and non-enzymatic oxidation processes, these PUFAs are transformed into a variety of oxylipins. They can be generated from the different precursor PUFAs by three main classes of enzymes - cyclooxygenase (COX), lipoxygenase (LOX), and cytochrome P450 (CYP450) - and thus form a complex pool of bioactive components (Fig. 1). Prostaglandins (PG) and thromboxanes (TX) are generated via the initial oxidation through COX pathways [4]. Prostanoids derived from AA are generally known as class 2 PGs and TXs due to their residual double-bond number, e.g., $\mathrm{PGE}_{2}$ and $\mathrm{TXB}_{2}$. Class 1 and 3 PGs and TX are similarly produced through the oxidation of DGLA and EPA, respectively [5]. Leukotrienes, likewise involved in pro-inflammatory signaling, especially in chemotaxis of leukocytes, are generated via the LOX pathways $[4,6]$. In mammalian cells, three LOX hydroperoxidases families exist, 5-LOX, 15-LOX, and 12-LOX, from which 5-LOX generates leukotrienes. Other LOX-dependent products include the mid-chain alcohols such as the AA-derived hydroxyeicosatetraenoic acids (HETEs), as well as the LAderived hydroxyoctadecadienoic acids (HODEs) and EPAderived hydroxypentaenoic acids (HEPEs). HETEs are known to be bioactive lipid mediators in chemotaxis and degranulation processes of neutrophils [7-9]. Omega-terminal oxidation of these PUFAs by CYP450 yields additional metabolites such as epoxides, diols, and further HETEs. Epoxides derived from AA are known to be associated to cardiovascular functions, in particular acting as vasodilators of human coronary arteries $[10,11]$. For epoxides derived from EPA, similar functions are evident [12]. While conversion of the epoxy fatty acids to diols reduces their vasoactive properties, the diols themselves appear to have distinct functions, for instance as ligands of the peroxisome proliferator activated receptor alpha subtype $(\operatorname{PPAR} \alpha)[13,14]$.

Although several oxylipins have been allocated to regulatory functions, the biological impact for many others remains unclear. Therefore, the bioactive potential of the less wellcharacterized lipids, either as unique functions or interaction with structural analogs from different precursor PUFAs, is likely to provide a rich source of novel insights into the regulation of mammalian responses to disease and tissue injury. Covering a wide range of these highly bioactive compounds still remains a challenge with modern analytical techniques. As a common approach, immunoassays are performed, either as radioimmunoassay [15, 16], enzyme immunoassays [17], or luminescent immunoassays $[18,19]$. They are specific and focus on one or only few compounds. Gas chromatography coupled to mass spectrometry (GC-MS) technology provides a sensitive methodology for a broad range of oxylipins [20]. However, to increase volatility, derivatization steps are essential. To avoid additional modifications, liquid chromatographymass spectrometric (LC-MS) techniques are one of the most sensitive and specific tools to simultaneously study many analogous analytes. Numerous LC-MS/MS methods have been described in literature for identification and quantification of low abundant oxylipins using multiple reaction monitoring (MRM) as an ion selective mode. Many methods focus on certain component classes of oxylipins as for instance prostaglandins [21-23], non-prostanoid eicosanoids [24], COX and LOX derived eicosanoids [25, 26], CYP450 related compounds $[27,28]$, and AA derived lipid mediators including metabolites of all three pathways [29-31]. However, the bioactive lipids of different origins are produced within the same cascade and cross-linked in a complex regulatory network (Fig. 1). Thus, the complex combination of chemically and structurally related oxylipins derived from the different PUFAs has become a challenge in modern LC-MS/MS techniques [32-36]. The development of a generic analytical oxylipin platform, intended for the application to a variety of biological matrices, will provide valuable information on metabolites derived from different precursor PUFAs and contribute importantly to our knowledge on inflammation processes.

Within this work we expand these methods to include an even broader array of prostanoids while demonstrating the utility of dynamic multiple reaction monitoring (dMRM) mode to focus on short retention time windows and enhance sensitivity. We applied our platform to human plasma derived from patients a day before and $24 \mathrm{~h}$ after cardiac surgery. Previous studies have demonstrated involvement of eicosanoids in myocardial ischemia/reperfusion injury. Arachidonic acid accumulates in the reperfused heart after an ischemic period and levels of prominent eicosanoids like $\mathrm{PGE}_{2}, \mathrm{TXB}_{2}$, and 6-keto-PGF $1 \alpha$ 
Fig. 1 Biosynthesis of oxylipins derived from linoleic acid (LA; green), dihomo- $\gamma$ linolenic acid (DGLA; red), arachidonic acid (AA; blue), eicosapentaenoic acid (EPA; orange), docosahexaenoic acid (DHA; yellow), and $\alpha$-linolenic acid (ALA; purple) via lipoxygenase (LOX), cyclooxygenase (COX), and cytochrome P450 (CYP450) pathways; $\mathrm{PLA}_{2}$ : phospholipase $\mathrm{A}_{2}$; metabolites (bold marked) are included in the oxylipin library of the described analytical platform

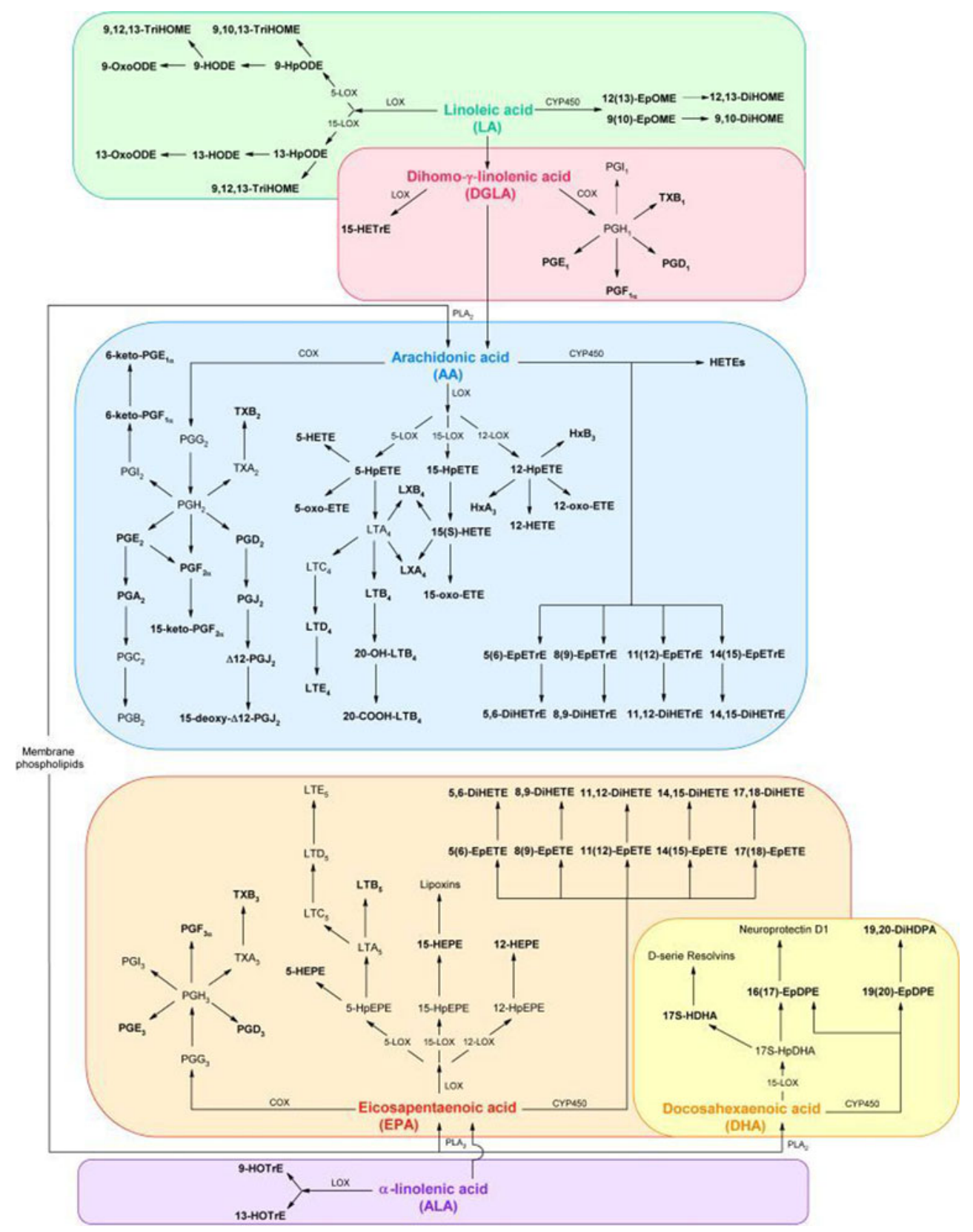

change rapidly after reperfusion [37-39]. In this study, we focus on late phase (i.e., $24 \mathrm{~h}$ post surgery) changes in circulating eicosanoids and further demonstrate the applicability of this generic LC-MS/MS platform to monitor physiological levels of a broad range of eicosanoids and related metabolites derived from different PUFAs in human plasma.

\section{Material and methods}

Materials

Ultra performance liquid chromatography (UPLC)-grade acetonitrile, isopropanol, methanol, ethyl-acetate, and water were purchased from Biosolve (the Netherlands). Glacial acetic acid was from Sigma-Aldrich (St. Louis, MO). High performance liquid chromatography (HPLC) was performed with the Ascentis Express C18 $(2.1 \times 150 \mathrm{~mm}, 2.7 \mu \mathrm{m})$ column from SigmaAldrich (St. Louis, MO). Solid phase extraction (SPE) was accomplished with Oasis HLB (60 mg/30 $\mu \mathrm{m})$ cartridges from Waters (Milford, MA). Deuterated and non-deuterated oxylipin standards were purchased either from Cayman Chemicals (Ann Arbor, MI), Biomol (Plymouth Meeting, PA), or Larodan (Malmö, Sweden). Human EDTA-plasma for method validation was provided by Richmond Pharmacology Ltd. (London, UK). 
Patient population

Oxylipin profiling was performed on EDTA-plasma derived from patients before and after cardiac surgery. The study protocols were approved by the local ethics committee, and informed consent was obtained from each patient. All patients were male and with good left ventricular function and $55( \pm 5.4)$ years of age with a BMI of $27.1( \pm 4.1) \mathrm{kg} / \mathrm{m}^{2}$. As patients develop a state of inflammation upon cardiac surgery, these patients provided a well controlled sample set to monitor levels of oxylipins in their function as bioactive lipid mediators after cardiac surgery. During cardiac arrest (myocardial ischemia), cardiac surgery was executed with use of a heart-lung machine. After $124.2( \pm 21.9)$ min of ischemia, the aortic cross-clamp was removed to restore cardiac blood flow (i.e., reperfusion).

To avoid any effects of heparin, such as increased levels of oxylipins by heparin induced phopholipase A2 activity, described in literature [40, 41], samples collected during the period in which heparin was administered to the patient were not used. Heparin administrated in conjunction with heart-lung machine use is neutralized by protamine sulfate, administrated in the operating room directly after the surgical intervention. Based on our own confirmation of a heparin-dependent elevation in circulating oxylipins (data not shown) and their response to protamine sulfate treatment, we chose to analyze samples taken the day before surgery (no heparin administrated to the patient) and $24 \mathrm{~h}$ after reperfusion of the heart, when no influence of heparin could be detected. All baseline and $24 \mathrm{~h}$ post-reperfusion samples were taken at the same moment during the day, between 12:00 noon and 2:00 p.m., to reduce the potential impact of circadian rhythm-dependent cycling which can affect several oxylipins. While they show a morning peak, the concentrations reduce during the day [42-44].

\section{Oxylipin extraction from human blood plasma}

The samples $(10 \mathrm{~mL})$ were taken the day before surgery by venipuncture (baseline) from the brachial vein and $24 \mathrm{~h}$ after reperfusion from the radial artery. All samples were collected in pre-cooled tubes containing EDTA (BD Vacutainer, Plymouth, UK) and placed on melting ice immediately. Blood samples were centrifuged $\left(1.550 \times \mathrm{g}, 10 \mathrm{~min}, 4{ }^{\circ} \mathrm{C}\right)$ and the derived plasma supernatant was re-centrifuged $\left(10.000 \times g, 4 \mathrm{~min}, 4{ }^{\circ} \mathrm{C}\right)$ to obtain leukocyte and thrombocyte poor plasma. Aliquots were stored at $-80{ }^{\circ} \mathrm{C}$ until extraction. The extraction was performed as described previously [36]. For oxylipin analysis $250 \mu \mathrm{L}$ aliquots were taken. After thawing on ice, the samples were treated immediately with antioxidants (0.2 mg BHT/EDTA) and spiked with $5 \mu \mathrm{L}$ of internal standards (ISTDs) of a concentration of $1,000 \mathrm{nM}$, resulting in $100 \mathrm{nM}$ after reconstitution which is within the linear range of the method. Compound extraction was performed with solid phase extraction using Oasis HLB $(60 \mathrm{mg} / 30 \mu \mathrm{m})$. Oxylipins were eluted with $2 \mathrm{~mL}$ ethyl acetate after wetting the cartridge with $0.5 \mathrm{~mL}$ methanol. In contrast to Shearer and coauthors [36], the eluent was reduced under nitrogen stream instead under vacuum. The dried extract was subsequently reconstituted in $50 \mu \mathrm{L}$ solution of methanol and acetonitrile (1:1) containing $100 \mathrm{nM}$ 1-cyclohexyluriedo-3-dodecanoic acid (CUDA) as a quality marker for the analysis. Afterwards, the extract was filtered by centrifugation using Amicon Ultrafree-MC Durapore PVDF filter (pore-size $0.1 \mu \mathrm{M}$; Millipore, Bedford, MA).

Liquid chromatography and mass spectrometry

Samples were analyzed by liquid chromatography (Agilent 1260 , San Jose, CA, USA) coupled to electrospray ionization on a triple quadrupole mass spectrometer (Agilent 6460, San Jose, CA, USA). For analysis $5 \mu \mathrm{L}$ of the extract was injected. The auto sampler was cooled at $10{ }^{\circ} \mathrm{C}$. Chromatographic separation was achieved on an Ascentis Express $(2.1 \times 150$ $\mathrm{mm}, 2.7 \mu \mathrm{m}$ particles; Sigma-Aldrich Supelco) column using a flow rate of $0.35 \mathrm{~mL} / \mathrm{min}$ at $40{ }^{\circ} \mathrm{C}$ during a 26 min gradient (0-3.5 min from $15 \%$ B to $33 \% \mathrm{~B}, 3.5-5.5 \mathrm{~min} \mathrm{~B}$ to $38 \%$, 5-7 $\min$ to $42 \% \mathrm{~B}, 7-9 \mathrm{~min}$ to $48 \% \mathrm{~B}, 9-15 \min$ to $65 \% \mathrm{~B}$, $15-17 \mathrm{~min}$ to $75 \% \mathrm{~B}, 17-18.5 \mathrm{~min}$ to $85 \% \mathrm{~B}, 18.5-19.5 \mathrm{~min}$ to $95 \% \mathrm{~B}$, from 19.5 to $21 \mathrm{~min}$ to $15 \% \mathrm{~B}, 21-26 \min 15 \%$ B), while using the solvents A, $0.1 \%$ acetic acid, and B, 90:10 $v / v$ acetonitril/isopropanol. Electrospray ionization was performed in the negative ion mode using $\mathrm{N}_{2}$ at a pressure of $35 \mathrm{psi}$ for the nebulizer with a flow of $10 \mathrm{~L} / \mathrm{min}$ and a temperature of $300{ }^{\circ} \mathrm{C}$, respectively. The sheath gas temperature was $350^{\circ} \mathrm{C}$ with a flow rate of $11 \mathrm{~L} / \mathrm{min}$. The capillary was set at 3,500 V and the nozzle voltage was $1,000 \mathrm{~V}$.

To detect the individual oxylipins, MRM in negative ion mode was performed with individually optimized fragmentor voltage and collision energies (Optimizer application, MassHunter, Agilent). MRM transitions were achieved by flow injection of pure standards and the optimizer application and were compared to literature when available for the certain compounds. The detailed list of MRM transitions can be found in the Electronic Supplementary Material Table S-1. Instead of defining certain time segments, with fixed dwell times per compound, dynamic MRM was used, assuring optimal dwell time and sufficient data points per peak.

\section{Data preprocessing}

Peak determination and peak area integration was performed with Mass Hunter Quan (Agilent, Version B.04.00) while auto-integration was manually inspected and corrected if necessary. The obtained peak areas of targets were corrected 
by appropriate internal standards (ISTD) and calculated response ratios were used throughout the analysis.

\section{Method validation}

The method validation was performed as in-house 3-day protocol to determine linearity, LOD and LOQ, inter- and intra-day variation, reproducibility, matrix effect, and recovery for all compounds. Compound stability was not included in this validation, but is being performed in a dedicated experiment and currently ongoing (stability period up to 48 weeks at $-80{ }^{\circ} \mathrm{C}$ without antioxidants). Preliminary data on this stability experiment showed no significant degradation over a period of 6 month (data not shown). We spiked pooled EDTA-plasma (Richmond Pharmacology, London, UK) with the purchased standard compounds in different concentrations varying from a minimum of $2 \mathrm{nM}$ up to $558 \mathrm{nM}$ in 8 levels (corresponding to levels of 3 to $1,260 \mathrm{pg}$; Electronic Supplementary Material Table S-2) to determine the linearity. Each calibration level was extracted twice and injected three times. Calibration curves were calculated by linear regression with $1 / \mathrm{S}^{2}$ weighing. Not all levels were included for each analyte. Only if a response was measured, i.e., for many analytes the low levels were not included. Furthermore, if the endogenous concentration was relatively high compared to the additionally spiked concentration, there is no difference in measured response when going to the next spiked level. For the calibration line, only levels were included if a significant response increase occurred from level to level. The responses were compared by means of $t$ tests going from low to high level. For example, if the tests show no significant differences between level C0 compared to level $\mathrm{C} 1$ and $\mathrm{C} 1$ versus $\mathrm{C} 2$ but a significantly higher response for $\mathrm{C} 3$ compared to $\mathrm{C} 2$ than the measurements for $\mathrm{C} 0$ and $\mathrm{C} 1$ were not included in the calibration line and $\mathrm{C} 2$ was the lowest included level. LOD and LOQ were determined by signal to noise ratio $(\mathrm{S} / \mathrm{N})$ higher 3 and 10 , respectively. For the calculation of possible batch-to-batch effect, we compared three different concentrations, lower level (C3), middle (C5), and high level (C7). Three different batches were prepared at three following days. To determine the recovery of the oxylipins, we spiked the pooled plasma with 11 deuterated ISTDs prior to the extraction step and in parallel after the last step of the extraction procedure. The labeled standards are non-endogenous and can be distinguished from endogenous ones with mass spectrometry. The 11 chosen ISTDs were considered to be representative for the different compound classes. Recovery was calculated as Area $_{\text {spiked before }} /$ Area $_{\text {spiked after. }}$

\section{Results and discussion}

A robust and sensitive quantitative method for profiling oxylipins such as eicosanoids and related lipid mediators biosynthesized from AA and other PUFAs was developed. As the majority of these compounds are present in plasma at low concentrations, their detection and quantification require methods with high sensitivity. Our LC-MS/MS method, employing dynamic MRM (dMRM), allows the evaluation of more than 100 oxylipins in a targeted approach (Fig. 2). The performance of dMRM allows the triple

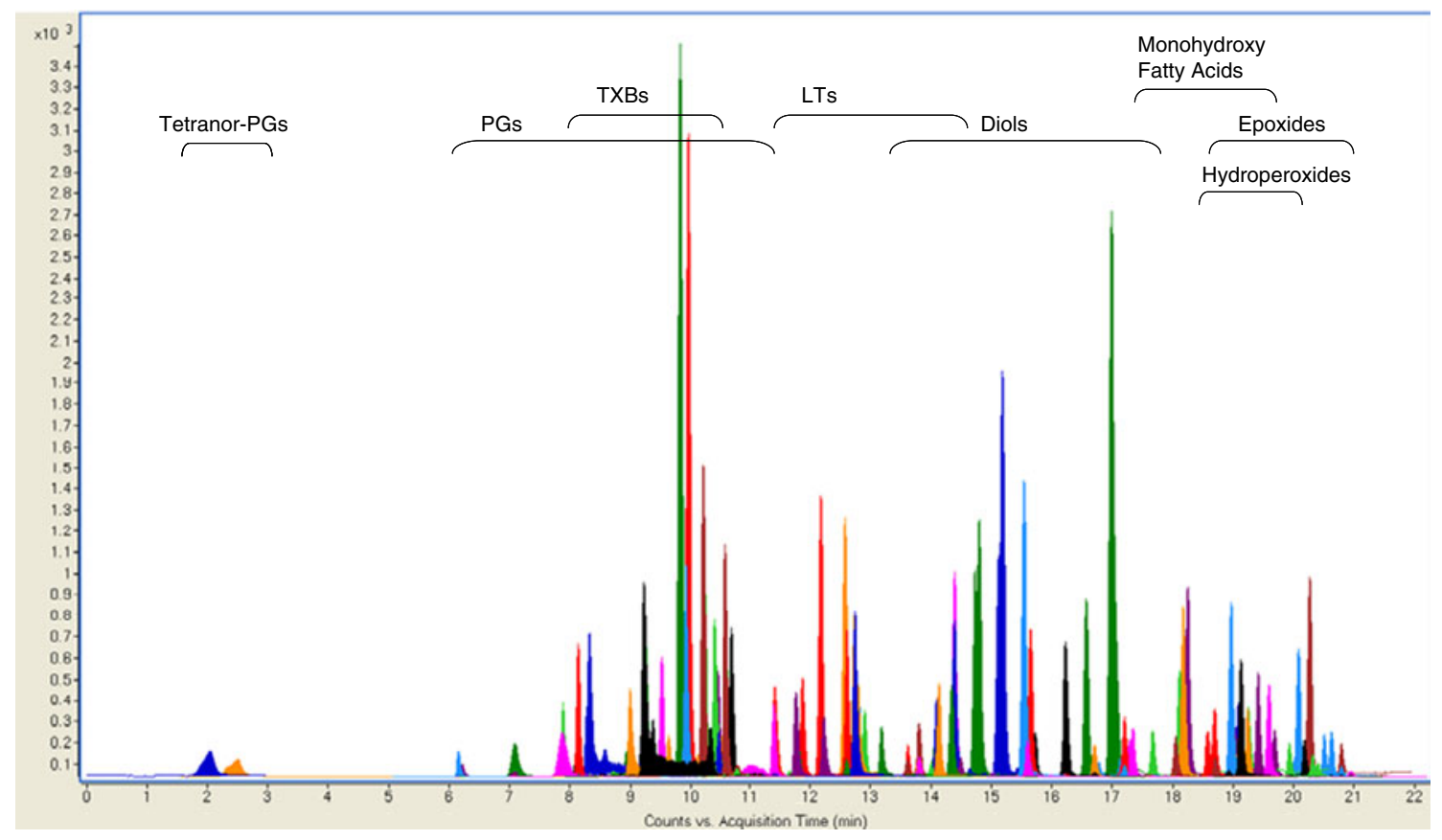

Fig. 2 LC-MS/MS chromatogram of 104 oxylipins performed on a triple quadrupole employing dynamic MRM in negative mode (exp. details; see the "Materials and methods" section and Table 2) 
quadrupole system to be focused directly on the expected analyte retention time $\left(t_{\mathrm{R}}\right)$ with a defined detection window instead of user-defined time segments to capture groups of closely eluting compounds. Allowing a constant cycle time for each transition, the dMRM procedure improves peak symmetry. Moreover, since transitions are only monitored in the defined detection windows in contrast to the whole time period of a standard MRM time segment, the rate of false positive detection (i.e., peak misidentification) is diminished. This is especially relevant for oxylipin families, which encompasses many isomeric and isobaric compounds. Furthermore, sensitivity can be enhanced since optimal dwell times can be achieved by reducing overlapping ion transitions [45].

For each compound, optimal transitions were determined to assure optimal peak detection (Electronic Supplementary Material Fig. S-1 and Table S-1). In the case of coeluting metabolites, compound specific precursor ions and their corresponding fragment ions allowed separation for selective detection and quantification of those compounds (Fig. 3). For instance, for 2,3-dinor-11ß-PGF ${ }_{2 \alpha}(\mathrm{m} / \mathrm{z} 325 \rightarrow$ $145)$ and 6-keto-PGF ${ }_{1 \alpha}(m / z 369 \rightarrow 163)$, both elute at $t_{\mathrm{R}}$ 7.89 min. Additional pairs were $\mathrm{PGF}_{1 \alpha} / 9,10,13$-TriHOME; 13,14-dihydro-15-keto-PGF ${ }_{1 \alpha} / 1 \mathrm{a}, 1$ b-dihomo-PGF $2 \alpha$; 8,15-DiHETE/12,13-DiHODE; 5,6-DiHETE/20-HETE; 13-HpODE/17(18)-EpETE; and 9-KODE/14(15)-EpETE (Fig. 3). For co-eluting isobars a unique fragment ion was chosen, as for 12-HETE $(\mathrm{m} / \mathrm{z} 319 \rightarrow 179)$ and 8 -HETE $(\mathrm{m} / \mathrm{z}$ $319 \rightarrow 115$ ), both eluting at $t_{\mathrm{R}} 19.24$ (Fig. 3). However, some critical pairs could not be separated spectrally with the current method. Those compounds were 19-hydroxy$\mathrm{PGE}_{2} / 20$-hydroxy-PGE 2 , 19-hydroxy- $\mathrm{PGF}_{2 \alpha} / 20$-hydroxy$\mathrm{PGF}_{2 \alpha}$, as well as $\mathrm{LTB}_{4} / 12$-epi $\mathrm{LTB}_{4}$ and 6-trans-LTB $/$ /6trans-12-epi $\mathrm{LTB}_{4}$. For detection and data evaluation, these compounds are therefore reported as combined amounts. For the hydroperoxides (HpETEs and HpODEs), we perceived a loss of an $\mathrm{H}_{2} \mathrm{O}$ molecule during ionization leading to $\left[\mathrm{M}-\mathrm{H}-\mathrm{H}_{2} \mathrm{O}\right]^{-}$. The water loss, especially for the hydroperoxides from AA, was also described previously [46].

Taken together, by employing dMRM and the detection of specified transitions in our HPLC-MS/MS approach, we were able to detect more than 100 different oxylipins.

\section{Method validation}

Validation measurements were performed using pooled EDTA-plasma as a sample matrix. Linearity, sensitivity (LOD and LOQ), reproducibility, matrix effect, and recovery were determined (Table 1 and Electronic Supplementary Material Table S-3).

Linearity and sensitivity The matrix samples (pooled plasma) were spiked with academic standards (eight concentrations).
We determined the linearity, LOD, and LOQ of each compound (104 targets). For the majority of the analytes, the $R^{2}$ ranged from 0.991 to 0.999 , followed by $0.981-0.99$ for $26 \%$ and $>0.9$ for $17 \%$ (Electronic Supplementary Material Table S-3). The range of LOQ was between 0.3 and $102 \mathrm{nM}$. For the majority of the targets ( $81 \%$ ), the LOQ was below $10 \mathrm{nM}$, for $56 \%$ it was below $3 \mathrm{nM}$. Relatively high LOQs were observed for 5,6-Lipoxin A4 with values higher than $80 \mathrm{nM}$.

Reproducibility To examine reproducibility, intra- and interbatch variability was assessed for all academic standards. A total of three batches were processed. In each batch duplicate extractions and triplicate injections were performed. In Table 1, oxylipins detected in human plasma are shown. The reproducibility values for all compounds are summarized in the Electronic Supplementary Material (Table S-3). For intra-batch effects (precision), the highest RSD value above the LOD was taken. For the oxylipins, the RSD values ranged from $4 \%$ up to $15 \%$ with exceptions for 20-HETE (41\%), 9,12,13-TriHOME (32\%), and 16(17)-EpDPE ( $21 \%)$. The batch-to-batch effects were higher for the low concentration level, compared to the high concentration level for the oxylipin platform. As problematic compounds with batch-to-batch effects higher than $50 \%, 15$-keto $\mathrm{PGF}_{1 \alpha}, \mathrm{PGK}_{2}$, Hepoxilin $\mathrm{A}_{3}, 5,6-$ Lipoxin $\mathrm{A}_{4}, \mathrm{LTE}_{4}$, 5HpETE, 12-HpETE, 15-HpETE, 9-HpODE, 13-HpODE, and $\mathrm{PGF}_{3 \alpha}$ need to be noted (Electronic Supplementary Material Table S-3).

Matrix effect Matrix effect was evaluated by comparing extracts of spiked matrix with extracts of spiked blank matrix using water as blank matrix sample. Due to irreversible adsorption during SPE of the standards from water as compared to plasma, extreme low values (approximately $10 \%$ ) of the expected values were observed, prohibiting this approach to calculate matrix effects. Since water is not suitable as blank matrix, matrix variation tests are recommended before application to the various matrices. Although no matrix effect was determined, final concentrations were not effected as the calibration lines were made in matrix as well.

Recovery For the recovery calculations, a set of deuterated ISTDs was used to prevent endogenous influences. Therefore, the standards were spiked either before or after the extraction and ratios of the peak areas were calculated. Recoveries ranged from $84 \%( \pm 4.4)$ to $45 \%( \pm 5.8)$, depending on the compound (Fig. 4). The recoveries for monohydroxy compounds (i.e., HETEs and HODEs) were lower than those of more polar prostaglandins and thromboxane. For (d3) $\mathrm{LTE}_{4}$, the recovery was lower than $60 \%$. In contrast to the other selected oxylipins, $\mathrm{LTE}_{4}$ contains a cysteinyl group, and like LTDs and LTCs, it belongs to the cysteinyl leukotriens. While recoveries differed between chemical class, inter-batch 
Fig. 3 LC-MS/MS extracted chromatograms of co-eluting oxylipins acquired by dynamic MRM. Retention time and specific MRM transitions are shown for each oxylipin

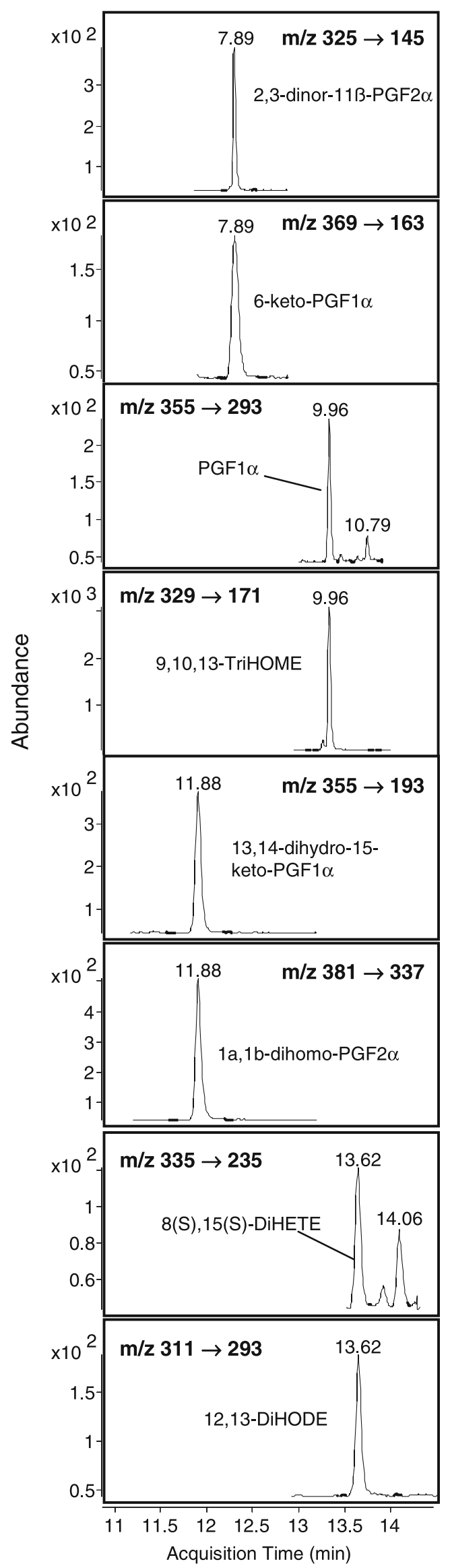


Table 1 Validation results of, in study samples, detected oxylipins

\begin{tabular}{|c|c|c|c|c|c|c|}
\hline Compound name & Retention time & $R^{2}$ & $\mathrm{LOD}[\mathrm{nM}]$ & LOQ [nM] & $\begin{array}{l}\text { Intra-batch effect } \\
\text { RSD [\%] }\end{array}$ & $\begin{array}{l}\text { Batch-to-batch effect } \\
\text { RSD [\%] }\end{array}$ \\
\hline
\end{tabular}

Arachidonic acid

\section{TXB2}

PGF $2 \alpha$

PGE2

$11 \beta$-PGE2

13,14-dihydro PGF2 $\alpha$

14,15-DiHETrE

11,12-DiHETrE

8,9-DiHETrE

5,6-DiHETrE

15-HETE

11-HETE

12-HETE

8-HETE

5-HETE

Linoleic acid

9,12,13-TriHOME

9,10,13-TriHOME

12,13-DiHOME

9,10-DiHOME

13-HODE

9-HODE

13-HpODE

13-KODE

9-HpODE

9-KODE

12(13)-EpOME

9(10)-EpOME

Dihomo-gamma-linolenic acid

PGF $1 \alpha$

15(S)-HETrE

9.94
19.41

0.996

0.988

0.8

0.5

0.967

0.3

1.1

0.990

0.993

4.7

0.8

15.5

0.983

0.985

0.9

17.67

18.05

0.983

0.3

0.1

15.60

19.93
0.991

0.988

1.0
2.9
0.6
3.1
38.2
1.0
1.7
1.0
1.2
0.8
0.9
0.6
4.7
0.6

1.0

3.1

38.2

1.0

1.7

1.0

1.2

0.8

0.9

0.6

4.7
0.6
0.8

1.8

1.0

1.8

13.9

0.5

11.8

2.4

29.1

10.8

8.4

1.9

2.5

1.7

Docosahexaenoic acid

19,20-DiHDPA

19(20)-EpDPE

$<14$

5-12

$<11$

7-21

$<32 \quad 9-16$

$<10 \quad 10-17$

$<4 \quad 3-6$

$<8 \quad 5-9$

$<9 \quad 7-16$

$<7 \quad 7-22$

$<6 \quad 23-72$

$<14 \quad 8-48$

$<7 \quad 29-72$

$<11 \quad 10-30$

$<7 \quad 14-39$

$<11 \quad 10-38$

19(20)-EpDE

The table gives an overview about the acquired validation parameter linearity $\left(R^{2}\right)$, sensitivity (LOD/LOQ) and reproducibility (precision and batch-to-batch effect). Validation data were achieved in pooled EDTA-plasma 


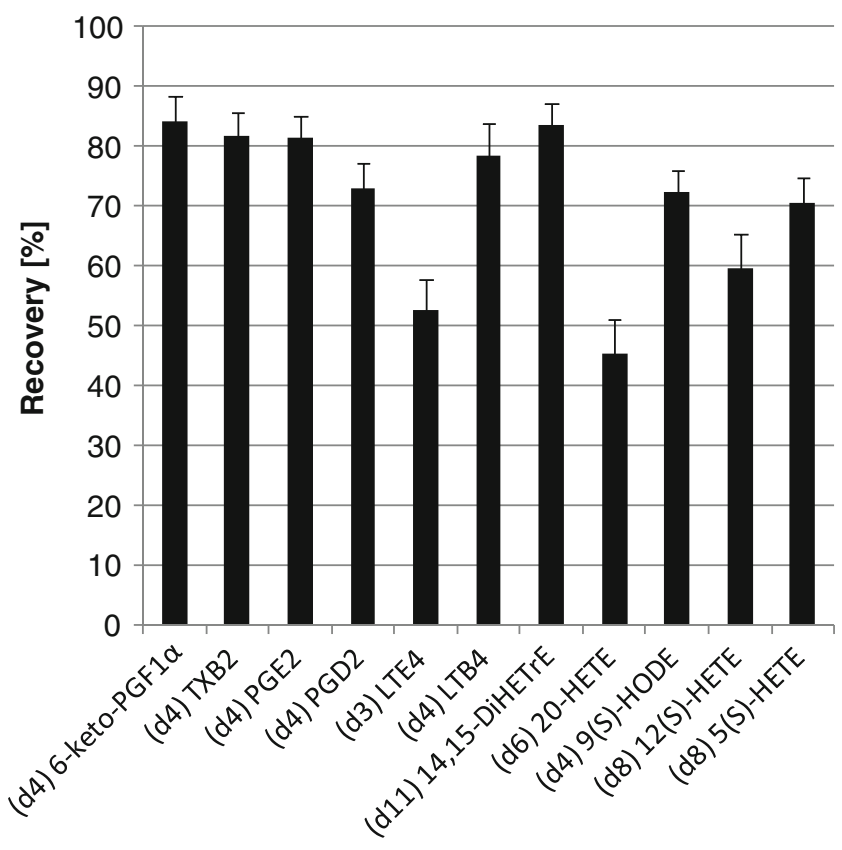

Fig. 4 Recovery of deuterated, non-endogenous oxylipins; the recovery is shown as percentage value; the compounds are arranged by their polarity, with the more polar ones on the left hand side

reproducibility was acceptable with RSD values between $4.3 \%$ and $9.6 \%$, except for (d3) LTE4 and (d6) 20-HETE with more than $10 \%(10.3 \%$ and $12.9 \%)$.

In summary, the analytical oxylipin platform developed here is characterized by high sensitivity reaching nanomolar levels, linearity with acceptable $R^{2}$, good reproducibility, and high coverage of oxylipin compounds.

\section{Detection of oxylipins in human plasma}

Applying the oxylipin profiling platform to human EDTAplasma, we were able to detect 36 oxylipins derived from 6 different PUFAs (Table 1). The majority of oxylipins detected in plasma are assigned to AA and LA, followed by metabolites derived from EPA, DHA, DGLA, and ALA. All these metabolites are biosynthesized via the three enzymatic COX, LOX, and CYP450 pathways. As the most prominent metabolites of the COX pathway, prostaglandins $\mathrm{PGE}_{2}$ and $\mathrm{PGF}_{2 \alpha}$ as well as the thromboxane $\mathrm{B}_{2}$, derived from AA, were detected. Furthermore, the prostaglandin $F_{1 \alpha}$ was detected, which is generated from DGLA as precursor, while no EPA derived 3 series prostanes were detected. The hydroxylated products of AA, LA, ALA, and DGLA represent a large target group in this analytical profile and are regulated by diverse sources in biological systems. For example, hydroxy lipids are generated in the LOX pathways (see Fig. 1), the COX pathways (e.g., LA metabolism by COX to HODEs [47, 48], as well as through non-enzymatic interactions of the PUFAs with reactive oxygen [49]. In addition, both epoxide and diol products from the CYP epoxygenase-dependent metabolism of AA (e.g., 11,12DiHETrE), LA (e.g., 9(10)-EpOME), EPA (e.g., 14,15DiHETE), and DHA (19(20)-EpDPE and 19,20-DiHPDA) were detected (Table 2).

In the previous studies, it was demonstrated that a variety of oxylipins could be detected in human plasma [35, 36, 50, 51]. Our findings are in agreement with these reported profiles. In our hands though, remarkable variations were observed in profiles and actual concentration levels between plasma pools of different origin, sample handling, and storage (data not shown).

Comparing patient oxylipin concentrations before surgery revealed substantial variation between patients. Previous studies have shown that even when individuals have the same physical condition, gender, and health, their basal oxylipin concentrations can differ remarkably [52]. Nevertheless, we observed similar patterns in abundance and relationships between certain compounds (Fig. 5). For instance, the levels of $\mathrm{PGF}_{2 \alpha}$ and its metabolite 13,14-dihydro- $\mathrm{PGF}_{2 \alpha}$ were consistently higher in their relative abundance than levels of $\mathrm{PGE}_{2}$ and its epimer $11 \beta-\mathrm{PGE}_{2}$. The levels of $\mathrm{TXB}_{2}$, the stable hydrolysis product of $\mathrm{TXA}_{2}$, were lower compared to the prostaglandins and thus indicate lower levels of $\mathrm{TXA}_{2}$. For CYP450 related compounds of the AA-pathway, 14,15-DiHETrE and 11,12-DiHETrE were found to be present at higher levels than 8,9-DiHETrE and 5,6-DiHETrE. Epoxides of the AA-pathway were below the detection limit and thus not detected in these samples. Also for the LA-pathway related oxylipins, a similarity in the basic abundance of the metabolites was observed. Although the epoxides and furthermore hydroperoxides originating from LA were detected above detection levels, they were less abundant compared to their subsequent derivatives.

\section{Alterations in oxylipin profiles during cardiac surgery}

All included patients were gender and age matched, and underwent the same surgical procedure. Twenty-four hours after surgery, the levels of CRP and IL-6 were significantly increased indicating an inflammatory response after the surgery (Fig. 6). The metabolic state of the AA pathway before and after cardiac surgery was monitored for each patient (Electronic Supplementary Material Fig. 2). Despite substantial inter-patient variation in oxylipin concentrations both before and after surgery, several consistent changes in the oxylipin profiles were observed. For the HETEs, compounds biosynthesized via the LOX pathway, a dramatic increase was visible, in particular for 12-HETE and 5-HETE (Fig. 7). The LOX products 12-HETE and 5-HETE are involved in chemotaxis of neutrophils [9, 53-55]. Activation and accumulation of neutrophils play key roles in inflammatory processes during ischemia-reperfusion injury [53, 56, 
Table 2 Oxylipins detected in human plasma

\begin{tabular}{|c|c|c|c|c|c|}
\hline LA & DGLA & AA & ALA & EPA & DHA \\
\hline \multicolumn{6}{|c|}{ Prostanoids/thromboids } \\
\hline & \multirow[t]{5}{*}{ PGF1a } & TXB2 & & & \\
\hline & & PGF2a & & & \\
\hline & & PGE2 & & & \\
\hline & & 11b-PGE2 & & & \\
\hline & & 13,14-dihydro-PGF2a & & & \\
\hline \multicolumn{6}{|l|}{ Diols } \\
\hline \multicolumn{2}{|l|}{ 12,13-DiHOME } & 14,15-DiHETrE & & 17,18-DiHETE & 19,20-DiHDPA \\
\hline \multirow[t]{4}{*}{ 9,10-DiHOME } & & $\begin{array}{l}\text { 11,12-DiHETrE } \\
\text { 8,9-DiHETrE }\end{array}$ & & 14,15-DiHETE & \\
\hline & & 5,6-DiHETrE & & & \\
\hline & & 5,15-DiHETE ${ }^{\mathrm{a}}$ & & & \\
\hline & & 8,15-DiHETE ${ }^{\mathrm{a}}$ & & & \\
\hline \multicolumn{6}{|l|}{ Epoxides } \\
\hline \multicolumn{2}{|l|}{ 12(13)-ЕpOME } & 14(15)-EpETrE ${ }^{\mathrm{a}}$ & & & 19(20)-EpDPE \\
\hline \multirow[t]{3}{*}{ 9(10)-EpOME } & & 11(12)-EpETrE ${ }^{\mathrm{a}}$ & & & $16(17)-\mathrm{EpDPE}^{\mathrm{a}}$ \\
\hline & & 8(9)-EpETrE & & & \\
\hline & & 5(6)-EpETrE ${ }^{\mathrm{a}}$ & & & \\
\hline \multicolumn{6}{|l|}{ Alcohols } \\
\hline & & 12-HETE & & 12-HEPE & \\
\hline \multicolumn{2}{|l|}{ 9-HODE } & 5-HETE & 9-HOTE & 5-HEPE & \\
\hline \multirow[t]{6}{*}{ 13-HODE } & 15-HETrE & 15-HETE & & 15-HEPE & 17-HDoHE \\
\hline & & 8-HETE & & & \\
\hline & & 9-HETE ${ }^{\mathrm{a}}$ & & & \\
\hline & & 20-HETE ${ }^{\mathrm{a}}$ & & & \\
\hline & & 11-HETE & & & \\
\hline & & 12-HHTrE ${ }^{\mathrm{a}}$ & & & \\
\hline \multicolumn{6}{|l|}{ Ketones } \\
\hline \multirow{2}{*}{$\begin{array}{l}\text { 13-KODE } \\
\text { 9-KODE }\end{array}$} & & $15-\mathrm{KETE}^{\mathrm{a}}$ & & & \\
\hline & & $5-\mathrm{KETE}^{\mathrm{a}}$ & & & \\
\hline \multicolumn{6}{|l|}{ Hydroperoxides } \\
\hline \multicolumn{2}{|l|}{ 13-HpODE } & $15-\mathrm{HpETE}^{\mathrm{a}}$ & & & \\
\hline \multicolumn{2}{|l|}{ 9-HpODE } & $5-\mathrm{HpETE}^{\mathrm{a}}$ & & & \\
\hline \multicolumn{6}{|l|}{ Triols } \\
\hline \multicolumn{6}{|l|}{ 9,12,13-TriHOME } \\
\hline 9,10,13-TriHOME & & & & & \\
\hline
\end{tabular}

Compounds are assigned to their specific precursor fatty acid

${ }^{\text {a }}$ Oxylipin metabolite was detected in pooled EDTA-plasma, but not in EDTA-plasma of the study samples

57]. Due to its chemotactic properties, 12-HETE has been linked to healing processes during inflammation $[58,59]$. Analysis of oxylipin profiles after abdominal aortic aneurism repair also showed high levels of 12-HETE in plasma $24 \mathrm{~h}$ after surgery for the group of patients which were in the resolution phase of inflammation [60].

Another 5-LOX generated compound $\mathrm{LTB}_{4}$ also promotes the chemotaxis of neutrophils $[4,6,53,56,57$, 


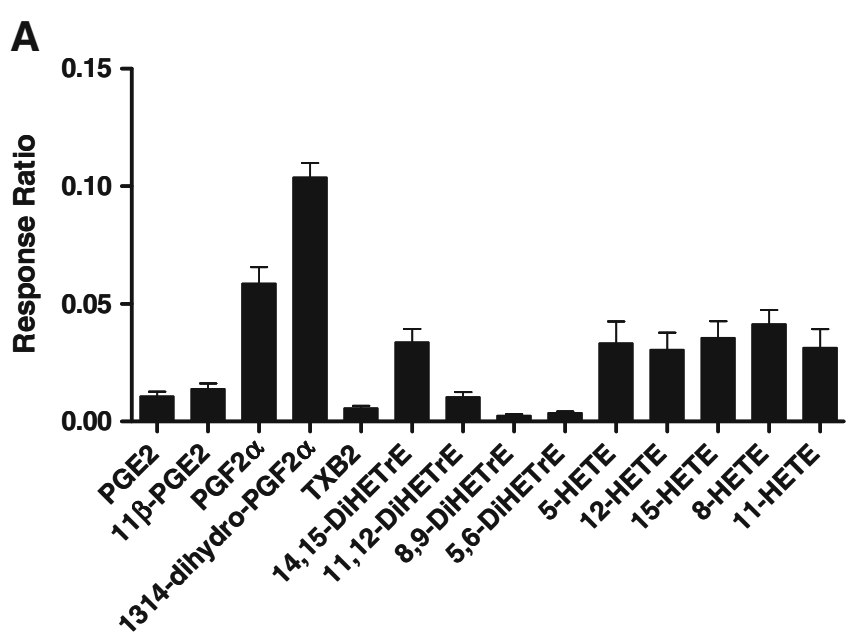

Fig. 5 Baseline level of oxylipins detected in patients undergoing cardiac surgery $(n=5)$; the majority of assigned oxylipins are from two pathways, (A) metabolites derived from arachidonic acid (AA)

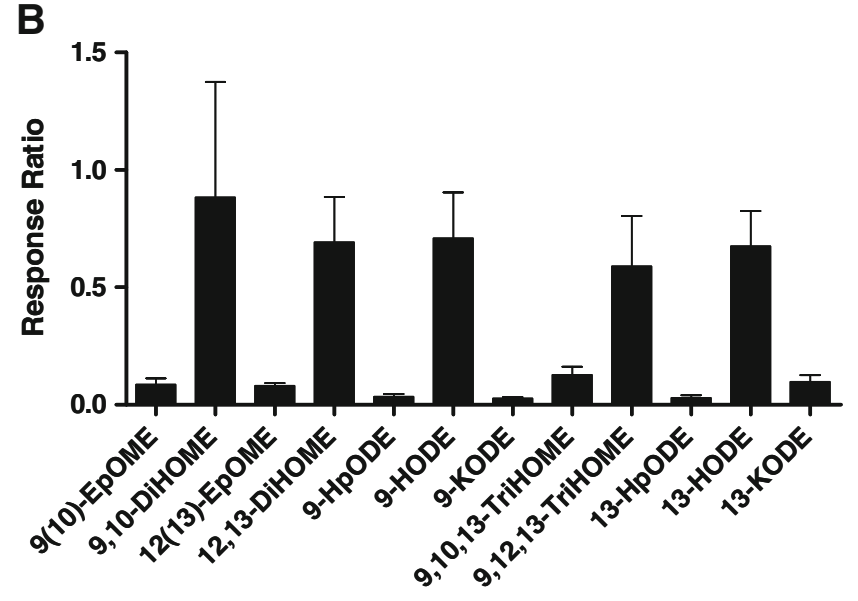

and (B) metabolites generated from linoleic acid (LA); each graph shows the relative abundance of oxylipins, plotted as relative response ratios (obtained abundance corrected by appropriated ISTDs)
Fig. 6 CRP- and IL-6 levels before and after surgery; (a) CRP levels $(n=5)$, after surgery the CRP levels increased dramatically; (b) IL-6 levels $(n=5)$, also for IL-6 levels a significant increment after surgery was observed
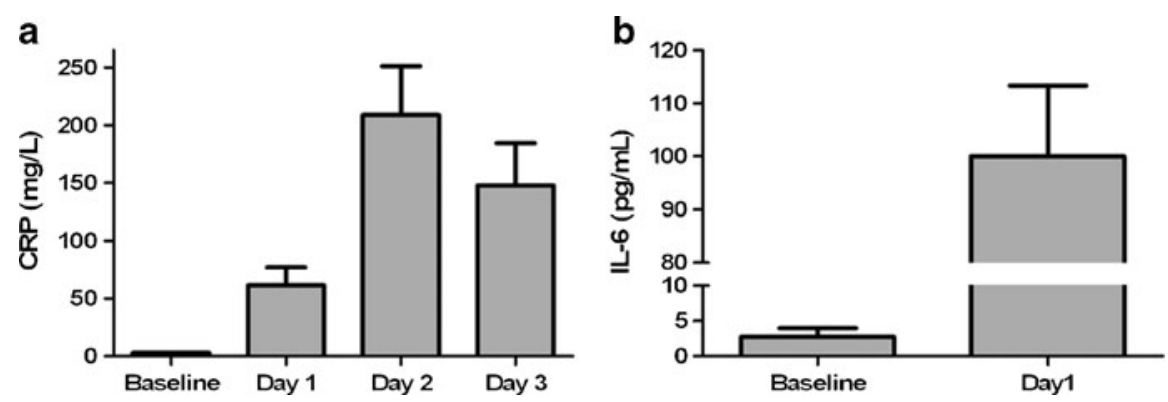

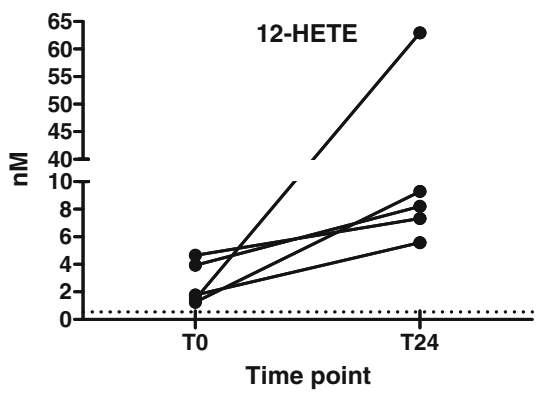

Fig. 7 The pairplot demonstrates the abundance of the two monohydroxy fatty acids 12-HETE and 5-HETE at baseline levels before the surgery (T0) and $24 \mathrm{~h}$ after the surgery (T24) of the patients $(n=5)$;

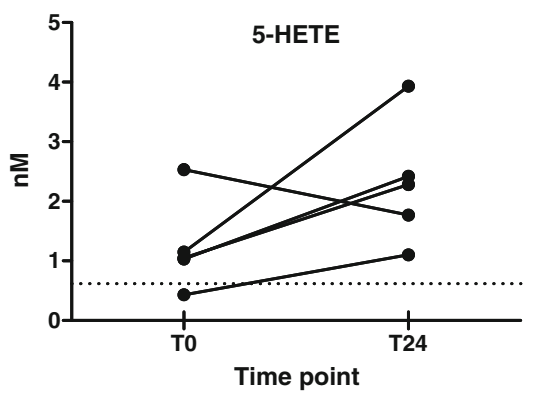

both oxylipins are enzymatically generated via lipoxygenase (12-LOX and 5-LOX, respectively) from arachidonic acid as precursor PUFA; dotted lines represent the limit of quantification 


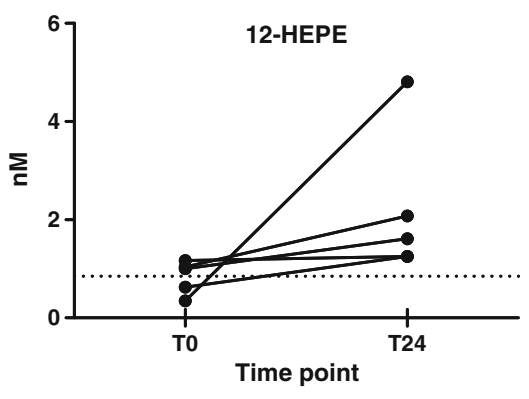

Fig. 8 The pairplot demonstrates the abundance of the two monohydroxy fatty acids 12-HEPE and 5-HEPE at baseline levels before the surgery (T0) and $24 \mathrm{~h}$ after the surgery (T24) of the patients $(n=5)$;

61-64]. Interestingly, no $\mathrm{LTB}_{4}$ was detected in plasma while 5-HETE was one of the metabolites increased after surgery. Besides the high levels of 12-HETE and 5-HETE, increases in the analogous EPA metabolites 12-HEPE and 5-HEPE were observed (Fig. 8). The metabolites 12-HEPE and 5HEPE are produced via the LOX pathway, but with EPA as precursor fatty acid. EPA derived LOX products can provide chemotactic activities, but are less potent than products of AA $[62,65]$.

For COX derived metabolites, such as the prostaglandins and thromboxanes, no significant changes were observed in human plasma (Electronic Supplementary Material Fig. 2). In previous studies on ischemia/reperfusion of the heart, metabolites of the COX pathway, especially $\mathrm{PGE}_{2}, \mathrm{TXB}_{2}$, and 6-keto-PGF $\mathrm{PG}_{1 \alpha}$, were significantly increased in heart perfusates [37]. A similar observation was made for CYP450 related compounds such as DiHETrEs. At the $24 \mathrm{~h}$ time point sampled here, only minor fluctuations were observed, suggesting acute COX-dependent responses had subsided by this time.

Our findings indicate that the analytical platform developed here allows the detection of consistent alterations in oxylipin profiles, even within a small patient population $(n=5)$.

\section{Conclusions}

We developed an LC-MS/MS method to detect oxylipins. Our target library comprises an expanded set of more than 100 compounds biosynthesized from six different PUFAs in one LC-MS/MS analysis. These targets include a wide array of both pro- and anti-inflammatory lipid mediators. Our oxylipin profiling platform was developed and validated for human plasma on a triple quadrupole mass spectrometer using multiple reaction monitoring operated in a dynamic MRM mode to guarantee high sensitivity, i.e., down to $\mathrm{nM}$ levels. This technology allows the sensitive detection of co-eluting compounds and isomers as well as several isobaric compounds by detecting specific transitions for each analyte with optimal dwell times in defined time windows. Our method was demonstrated

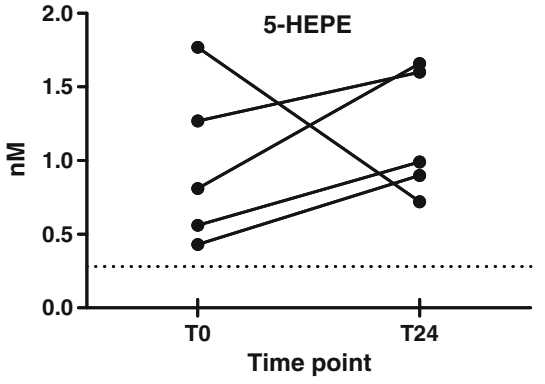

both are generated from eicosapentaenoic acid, biosynthesized by 12LOX and 5-LOX; dotted lines represent the lower limit of quantification

on patients undergoing cardiac surgery and was able to detect one third of our target library in human plasma. The metabolites 12-HETE and 5-HETE, known to be related to cardiac surgery and healing processes, were consistently increased after cardiac surgery. In addition, an increase of similar monohydroxy metabolites derived from EPA was observed. In conclusion, the analytical platform developed here allows specific and sensitive quantitative assessment of more than 100 oxylipins and may have wider applications in studies on the role of these highly bioactive metabolites in human diseases. The successful application of the developed method to this biological study implies that the developed platform can be used broadly to profile these highly bioactive compounds.

Acknowledgments This project was (co)financed by the Netherlands Metabolomics Centre (NMC) which is part of the Netherlands Genomics Initiative/Netherlands Organization for Scientific Research.

Open Access This article is distributed under the terms of the Creative Commons Attribution License which permits any use, distribution, and reproduction in any medium, provided the original author(s) and the source are credited.

\section{References}

1. Serhan CN, Savill J (2005) Resolution of inflammation: the beginning programs the end. Nat Immunol 6(12):1191-1197

2. Tapiero H, Ba GN, Couvreur P, Tew KD (2002) Polyunsaturated fatty acids (PUFA) and eicosanoids in human health and pathologies. Biomed Pharmacother 56(5):215-222

3. Fletcher JR (1993) Eicosanoids. Critical agents in the physiological process and cellular injury. Arch Surg 128(11):1192-1196

4. Funk CD (2001) Prostaglandins and leukotrienes: advances in eicosanoid biology. Science 294(5548):1871-1875

5. Bergstroem S, Samuelsson B (1965) Prostaglandins. Annu Rev Biochem 34:101-108

6. Bray MA, Ford-Hutchinson AW, Smith MJ (1981) Leukotriene B4: an inflammatory mediator in vivo. Prostaglandins 22(2):213-222

7. Bittleman DB, Casale TB (1995) 5-Hydroxyeicosatetraenoic acid (HETE)-induced neutrophil transcellular migration is dependent upon enantiomeric structure. Am J Respir Cell Mol Biol 12(3):260-267

8. Spector AA, Gordon JA, Moore SA (1988) Hydroxyeicosatetraenoic acids (HETEs). Prog Lipid Res 27(4):271-323 
9. Stenson WF, Parker CW (1980) Monohydroxyeicosatetraenoic acids (HETEs) induce degranulation of human neutrophils. J Immunol 124(5):2100-2104

10. Larsen BT, Miura H, Hatoum OA, Campbell WB, Hammock BD, Zeldin DC, Falck JR, Gutterman DD (2006) Epoxyeicosatrienoic and dihydroxyeicosatrienoic acids dilate human coronary arterioles via $\mathrm{BK}(\mathrm{Ca})$ channels: implications for soluble epoxide hydrolase inhibition. Am J Physiol Heart Circ Physiol 290(2):H491-499

11. Roman RJ (2002) P-450 metabolites of arachidonic acid in the control of cardiovascular function. Physiol Rev 82(1):131-185

12. Morin C, Sirois M, Echave V, Rizcallah E, Rousseau E (2009) Relaxing effects of 17(18)-EpETE on arterial and airway smooth muscles in human lung. Am J Physiol Lung Cell Mol Physiol 296 (1):L130-139

13. Newman JW, Morisseau C, Hammock BD (2005) Epoxide hydrolases: their roles and interactions with lipid metabolism. Progress in Lipid Research 44(1):1-51

14. Spector AA, Fang X, Snyder GD, Weintraub NL (2004) Epoxyeicosatrienoic acids (EETs): metabolism and biochemical function. Progress in Lipid Research 43(1):55-90

15. Dray F, Charbonnel B, Maclouf J (1975) Radioimmunoassay of prostaglandins Falpha, E1 and E2 in human plasma. Eur J Clin Invest 5(4):311-318

16. Jaffe BM, Behrman HR, Parker CW (1973) Radioimmunoassay measurement of prostaglandins $\mathrm{E}, \mathrm{A}$, and $\mathrm{F}$ in human plasma. $\mathrm{J}$ Clin Invest 52(2):398-405

17. Pradelles P, Grassi J, Maclouf J (1985) Enzyme immunoassays of eicosanoids using acetylcholine esterase as label: an alternative to radioimmunoassay. Anal Chem 57(7):1170-1173

18. Neupert W, Oelkers R, Brune K, Geisslinger G (1996) A new reliable chemiluminescence immunoassay (CLIA) for prostaglandin E2 using enhanced luminol as substrate. Prostaglandins 52(5):385-401

19. Wu C, Irie S, Yamamoto S, Ohmiya Y (2009) A bioluminescent enzyme immunoassay for prostaglandin $\mathrm{E}(2)$ using Cypridina luciferase. Luminescence 24(2):131-133

20. Tsikas D (1998) Application of gas chromatography-mass spectrometry and gas chromatography-tandem mass spectrometry to assess in vivo synthesis of prostaglandins, thromboxane, leukotrienes, isoprostanes and related compounds in humans. J Chromatogr B: Biomed Sci Appl 717(1-2):201-245

21. Cao H, Xiao L, Park G, Wang X, Azim AC, Christman JW, van Breemen RB (2008) An improved LC-MS/MS method for the quantification of prostaglandins $\mathrm{E}(2)$ and $\mathrm{D}(2)$ production in biological fluids. Anal Biochem 372(1):41-51

22. Masoodi M, Nicolaou A (2006) Lipidomic analysis of twentyseven prostanoids and isoprostanes by liquid chromatography/ electrospray tandem mass spectrometry. Rapid Commun Mass Spectrom 20(20):3023-3029

23. Takabatake M, Hishinuma T, Suzuki N, Chiba S, Tsukamoto H, Nakamura H, Saga T, Tomioka Y, Kurose A, Sawai T, Mizugaki M (2002) Simultaneous quantification of prostaglandins in human synovial cell-cultured medium using liquid chromatography/tandem mass spectrometry. Prostaglandins Leukot Essent Fatty Acids 67(1):51-56

24. Zhang JH, Pearson T, Matharoo-Ball B, Ortori CA, Warren AY, Khan R, Barrett DA (2007) Quantitative profiling of epoxyeicosatrienoic, hydroxyeicosatetraenoic, and dihydroxyeicosatetraenoic acids in human intrauterine tissues using liquid chromatography/electrospray ionization tandem mass spectrometry. Anal Biochem 365(1):40-51

25. Dahl SR, Kleiveland CR, Kassem M, Lea T, Lundanes E, Greibrokk $\mathrm{T}$ (2009) Determination of thromboxanes, leukotrienes and lipoxins using high-temperature capillary liquid chromatography-tandem mass spectrometry and on-line sample preparation. J Chromatogr A 1216(22):4648-4654

26. Schmelzer KR, Kubala L, Newman JW, Kim IH, Eiserich JP, Hammock BD (2005) Soluble epoxide hydrolase is a therapeutic target for acute inflammation. Proc Natl Acad Sci U S A 102 (28):9772-9777

27. Newman JW, Watanabe T, Hammock BD (2002) The simultaneous quantification of cytochrome $\mathrm{P} 450$ dependent linoleate and arachidonate metabolites in urine by HPLC-MS/MS. J Lipid Res 43 (9): $1563-1578$

28. Nithipatikom K, Grall AJ, Holmes BB, Harder DR, Falck JR, Campbell WB (2001) Liquid chromatographic-electrospray ionization-mass spectrometric analysis of cytochrome P450 metabolites of arachidonic acid. Anal Biochem 298(2):327-336

29. Deems R, Buczynski MW, Bowers-Gentry R, Harkewicz R, Dennis EA (2007) Detection and quantitation of eicosanoids via high performance liquid chromatography-electrospray ionization-mass spectrometry. Methods Enzymol 432:59-82

30. Mesaros C, Lee SH, Blair IA (2009) Targeted quantitative analysis of eicosanoid lipids in biological samples using liquid chromatography-tandem mass spectrometry. J Chromatogr B Analyt Technol Biomed Life Sci 877(26):2736-2745

31. Yue H, Jansen SA, Strauss KI, Borenstein MR, Barbe MF, Rossi LJ, Murphy E (2007) A liquid chromatography/mass spectrometric method for simultaneous analysis of arachidonic acid and its endogenous eicosanoid metabolites prostaglandins, dihydroxyeicosatrienoic acids, hydroxyeicosatetraenoic acids, and epoxyeicosatrienoic acids in rat brain tissue. J Pharm Biomed Anal 43 (3):1122-1134

32. Blaho VA, Buczynski MW, Brown CR, Dennis EA (2009) Lipidomic analysis of dynamic eicosanoid responses during the induction and resolution of Lyme arthritis. J Biol Chem 284 (32):21599-21612

33. Masoodi M, Mir AA, Petasis NA, Serhan CN, Nicolaou A (2008) Simultaneous lipidomic analysis of three families of bioactive lipid mediators leukotrienes, resolvins, protectins and related hydroxy-fatty acids by liquid chromatography/electrospray ionisation tandem mass spectrometry. Rapid Commun Mass Spectrom 22(2):75-83

34. Newman JW, Kaysen GA, Hammock BD, Shearer GC (2007) Proteinuria increases oxylipid concentrations in VLDL and HDL but not LDL particles in the rat. J Lipid Res 48(8):1792-1800

35. Psychogios N, Hau DD, Peng J, Guo AC, Mandal R, Bouatra S, Sinelnikov I, Krishnamurthy R, Eisner R, Gautam B, Young N, Xia J, Knox C, Dong E, Huang P, Hollander Z, Pedersen TL, Smith SR, Bamforth F, Greiner R, McManus B, Newman JW, Goodfriend T, Wishart DS (2011) The human serum metabolome. PLoS One 6(2):e16957

36. Shearer GC, Harris WS, Pedersen TL, Newman JW (2010) Detection of omega-3 oxylipins in human plasma and response to treatment with omega-3 acid ethyl esters. J Lipid Res 51 (8):2074-2081

37. Cordis GA, Das DK (1991) High-performance liquid chromatographic detection of myocardial prostaglandins and thromboxanes. J Chromatogr 536(1-2):309-317

38. Otani H, Prasad MR, Jones RM, Das DK (1989) Mechanism of membrane phospholipid degradation in ischemic-reperfused rat hearts. Am J Physiol 257(1 Pt 2):H252-258

39. Van der Vusse GJ, Reneman RS, van Bilsen M (1997) Accumulation of arachidonic acid in ischemic/reperfused cardiac tissue: possible causes and consequences. Prostaglandins Leukot Essent Fatty Acids 57(1):85-93

40. Goodfriend TL, Pedersen TL, Grekin RJ, Hammock BD, Ball DL, Vollmer A (2007) Heparin, lipoproteins, and oxygenated fatty acids in blood: a cautionary note. Prostaglandins Leukot Essent Fatty Acids 77(5-6):363-366

41. Nakamura H, Kim DK, Philbin DM, Peterson MB, Debros F, Koski G, Bonventre JV (1995) Heparin-enhanced plasma phospholipase A2 activity and prostacyclin synthesis in patients undergoing cardiac surgery. J Clin Invest 95(3):1062-1070 
42. Kurokawa K, Tanaka H, Tanaka S, Abe S (2001) Circadian characteristics of urinary leukotriene $\mathrm{E}(4)$ in healthy subjects and nocturnal asthmatic patients. Chest 120(6):1822-1828

43. Nadler JL, Yamamoto JV (1986) Diurnal variation and exercise induced changes of prostacyclin in man. Prostaglandins Leukot Med 22(1):71-78

44. Rigas B, Levine L (1983) Human salivary eicosanoids: circadian variation. Biochem Biophys Res Commun 115(1):201-205

45. Rajagopalan S., R. Gudihal, Waddell K (2010) Dynamic MRM: a clear advantage for high-throughput protein quantitation. Agilent Technologies, Inc 2010:5990-5092EN

46. MacMillan DK, Murphy RC (1995) Analysis of lipid hydroperoxides and long-chain conjugated keto acids by negative ion electrospray mass spectrometry. Journal of the American Society for Mass Spectrometry 6(12):1190-1201

47. Funk CD, Powell WS (1983) Metabolism of linoleic-acid by prostaglandin endoperoxide synthase from adult and fetal bloodvessels. Biochimica Et Biophysica Acta 754(1):57-71

48. Hamberg M, Samuelsson B (1980) Stereochemistry in the formation of 9-hydroxy-10,12-octadecadienoic acid and 13-hydroxy9,11-octadecadienoic acid from linoleic acid by fatty acid cyclooxygenase. Biochim Biophys Acta 617(3):545-547

49. Tanito M, Yoshida Y, Kaidzu S, Ohira A, Niki E (2006) Detection of lipid peroxidation in light-exposed mouse retina assessed by oxidative stress markers, total hydroxyoctadecadienoic acid and 8-iso-prostaglandin F2alpha. Neurosci Lett 398(1-2):63-68

50. Quehenberger O, Armando AM, Brown AH, Milne SB, Myers DS, Merrill AH, Bandyopadhyay S, Jones KN, Kelly S, Shaner RL, Sullards CM, Wang E, Murphy RC, Barkley RM, Leiker TJ, Raetz CR, Guan Z, Laird GM, Six DA, Russell DW, McDonald JG, Subramaniam S, Fahy E, Dennis EA (2010) Lipidomics reveals a remarkable diversity of lipids in human plasma. J Lipid Res 51 (11):3299-3305

51. Van Erk MJ, Wopereis S, Rubingh C, van Vliet T, Verheij E, Cnubben NH, Pedersen TL, Newman JW, Smilde AK, van der Greef J, Hendriks HF, van Ommen B (2010) Insight in modulation of inflammation in response to diclofenac intervention: a human intervention study. BMC Med Genomics 3:5

52. Helmersson J, Basu S (2006) Intra-day variation of in vivo prostaglandin F2alpha formation in healthy subjects. Prostaglandins Other Lipid Mediat 80(1-2):93-99
53. Entman ML, Michael L, Rossen RD, Dreyer WJ, Anderson DC, Taylor AA, Smith CW (1991) Inflammation in the course of early myocardial ischemia. FASEB J 5(11):2529-2537

54. Goetzl EJ, Brash AR, Tauber AI, Oates JA, Hubbard WC (1980) Modulation of human neutrophil function by monohydroxyeicosatetraenoic acids. Immunology 39(4):491-501

55. Lagarde M, Gualde N, Rigaud M (1989) Metabolic interactions between eicosanoids in blood and vascular cells. Biochem J 257 (2):313-320

56. Grace PA (1994) Ischaemia-reperfusion injury. Br J Surg 81 (5):637-647

57. Jordan JE, Zhao ZQ, Vinten-Johansen J (1999) The role of neutrophils in myocardial ischemia-reperfusion injury. Cardiovasc Res 43(4):860-878

58. Morykwas MJ, Duell EA, Jennings DA, Ledbetter MS, White WL, Argenta LC (1992) Arachidonic acid metabolites: effects on inflammation of fetal rabbit excisional wounds. Inflammation 16 (3):251-258

59. Van der Ham AC, Kort WJ, Bijma AM, Zijlstra FJ, Vermeer MA, Jeekel J (1990) Eicosanoid profile of healing colon anastomosis and peritoneal macrophages in the rat. Gut 31(7):807-811

60. Pillai P, Leeson S, Porter T, Owens C, Kim J, Conte M, Serhan C, Gelman S (2011) Chemical mediators of inflammation and resolution in post-operative abdominal aortic aneurysm patients. Inflammation. doi:2010.1007/s10753-10011-19294-10758

61. Buczynski MW, Dumlao DS, Dennis EA (2009) Thematic review series: proteomics. An integrated omics analysis of eicosanoid biology. J Lipid Res 50(6):1015-1038

62. Heidel JR, Taylor SM, Laegreid WW, Silflow RM, Liggitt HD, Leid RW (1989) In vivo chemotaxis of bovine neutrophils induced by 5 -lipoxygenase metabolites of arachidonic and eicosapentaenoic acid. Am J Pathol 134(3):671-676

63. Peters-Golden M, Brock TG (2000) Intracellular compartmentalization of leukotriene biosynthesis. Am J Respir Crit Care Med 161 (2 Pt 2):S36-40

64. Samuelsson B, Dahlen SE, Lindgren JA, Rouzer CA, Serhan CN (1987) Leukotrienes and lipoxins: structures, biosynthesis, and biological effects. Science 237(4819):1171-1176

65. Moreno JJ (2009) Differential effects of arachidonic and eicosapentaenoic Acid-derived eicosanoids on polymorphonuclear transmigration across endothelial cell cultures. J Pharmacol Exp Ther 331(3):1111-1117 